\title{
LET-756/FGF is Implicated in the Control of C. elegans Body Size
}

\author{
Cornel Popovici ${ }^{1,2}$, Sylvain Hiver $^{1}$, Daniel Birnbaum ${ }^{1}$ and Régine Roubin ${ }^{*}, 1$
}

\author{
${ }^{1}$ Inserm UMR891, Centre de Recherche en Cancérologie de Marseille; Institut Paoli-Calmettes, Université de la Médi- \\ terranée, 13009, Marseille, France \\ ${ }^{2}$ Present Address : Département de Génétique Médicale, Hôpital de la Timone - Enfants, Assistance Publique - Hôpi- \\ taux de Marseille, 13005, Marseille, France
}

\begin{abstract}
LET-756 is one of the two fibroblast growth factors (FGF) of Caenorhabditis elegans. We have previously shown that worms homozygote for the let-756 null allele (s2887) die at larval stage and that homozygotes for the partial loss-of-function allele $(s 2613)$ are viable but shorter than wild-type animals. We now show that expression of let-756 in any region of the $s 2887$ worm can rescue the lethal phenotype but with variations in body size depending on the site of expression, harmonious growth being achieved by let-756 expression in body wall muscles. Local action of let-756 was also observed in mosaic animals homozygotes for the $s 2613$ allele. Our results support a paracrine activity for LET-756 exerting its effect locally on body size whether by acting as an actual growth factor or by acting on EGL-15/FGFR expressing hypodermal cells thereby regulating fluid balance.
\end{abstract}

\section{INTRODUCTION}

In vertebrates, Fibroblast Growth Factors (FGFs) are involved in various developmental and pathological processes (for review see [1]), usually in combination with other regulatory growth factors, such as members of WNT and TGF superfamilies. In humans, 22 FGFs are distributed in seven families [2]. FGFs activate transmembrane FGF tyrosine kinase receptors (FGFR). Activated FGFRs dimerize, are phosphorylated, and signal through cytoplasmic proteins. Coreceptors, such as heparan sulphate proteoglycans, are required for proper membrane signaling. A special family of FGFs, the fibroblast homologous factors (FHF), has different functions and signaling pathways [3]. Some FGFs may signal through a direct nuclear pathway since FGFs or both FGFs and FGFRs have been identified in the nucleus (reviewed in [4]).

Only a few FGFs have been found in protostomian species whose sequences are available. In the worm Caenorhabditis elegans, two FGFs, EGL-17 and LET-756, and one FGFR, EGL-15, have been described [5-6; for review 7]. An EGL-17 mutant has a defect in migration of sex muscles, and consequently in vulva function and egg laying $[8,9]$. LET-756 is essential for worm development [10]. It regulates axonal growth [11], fluid homeostasis [12], muscle protein degradation [13] and muscle membrane extension [14].

In $C$. elegans, three different pathways determine body length: a TGF $\beta$ pathway, involving $d b l-1, s m a-2, s m a-3$, sma-4, lon-1 and lon-2 genes, a spectrin pathway involving sma-1, spc-1 and $u n c-70$ and a calcineurin pathway involving sensory neurons and muscle cells expressing tax- 6 and

*Address correspondence to this author at the Inserm UMR891, Centre de Recherche en Cancérologie de Marseille, Institut Paoli-Calmettes, Université de la Méditerranée, 13009, Marseille, France;

E-mail: regine.roubin@inserm.fr $c n b-1$ [15-19]. An underlying ectodermal cell layer called the hypodermis makes cuticle, an exoskeleton of collagenous extracellular matrix (ECM). The hypodermis surrounds the body of the animal [reviewed in 20]. Proteins implicated in body length act on or are expressed in hypodermal cells. Thus, the hypodermis is the primary site where growth is regulated.

LET-756/FGF partial loss-of-function mutants are of the same size as wild-type (wt) animals at hatching, but grow more slowly during larval stages and are about half the length of, and are thinner than wt worms in adulthood [10]. Reduction in body size could be due to reduced cell number, reduced cell size or fluid loss. Because no difference in the number of nuclei in wt and let-756 (s2613) mutants has been found [10], the small body size phenotype could be due to a reduction in size of some or all cells of the animal or to body fluid loss.

To document the effect of let-756 on body size we performed rescue experiments of both the partial loss-offunction allele (s2613) and the null allele (s2887), followed by a morphometric analysis of the body size of the rescued animals. To determine which site of expression was important for rescue, let-756 was expressed in various areas of the body.

\section{MATERIALS AND METHODOLOGY}

\section{Nematode Culture and Transformation Rescue Experiments}

C. elegans Bristol (N2) strain nematodes were cultured using standard techniques [21]. let-756 rescuing activity was assayed by injecting tester DNA at $50 \mathrm{ng}$ per $\mu 1$ into gravid $s 2887$ strain hermaphrodites, establishing transformed lines and scoring the Dpy progeny for a viable phenotype. The $s 2887$ allele used in these experiments was generated on a dpy-17(e164) unc-32(e189) chromosome after UV irradiation [22]; the $s D p 3$, a portion of chromosome III, balances the lethal phenotype of the $s 2887$ strain. The rescue activity was tested for the presence in the progeny of live Dpy ani- 
mals. Dpy animals were genotyped for the presence of the inversion in the let-756 locus characteristic of the $s 2887$ strain by PCR on single worm, as described in [23]. The strains $d p y-17(e 164)$ unc-32 (e189) and unc-32 (e189) used as control, were obtained from the Caenorhabditis Genetics Center (CGC).

The hypomorph FF628 let-756(s2613) unc-32(e189) strain was isolated by D. Thierry-Mieg from BC4253 and outcrossed an additional 7 times. Homozygous animals are viable and fertile, but slow growing, transparent and small. These animals were coinjected with $P_{\text {let-756: }}:$ let-756 and the pPD93.97 plasmid which contains $P_{\text {myo }}: \because G F P$. Fluorescent cells contain arrays bearing both plasmids and mosaic animals were analyzed.

\section{Fusion of Tissue Specific Promoters and let-756::gfp Coding Region}

The promoters used in muscle-specific expression of let$756:: g f p$ were myo-2 (pharyngeal muscles), myo-3 (body muscles) and egl-17 (sex muscles) [24, 8]. The constructs driving the coding region of let-756::gfp and let-756 $6^{R 318}:: g f p$ under the control of $P_{\text {let-756 }}$ promoter have already been reported [25]. The $P_{m y o-3}: \because$ let-756: $: g f p$ expression construct was made by ligating the PCR product containing the let-756 cDNA in the XbaI-BamHI digested pPD93.97. The XbaI$E c o$ RI fragment containing the let-756::gfp fusion was then excised and inserted in the NheI-BglII digested pPD96.48 vector to obtain $P_{\text {myo-2 }}: \because l e t-756: \because g f p$ expression construct. The full length let-756 genomic DNA under the control of egl-17 promoter (NH\#469) was a gift from M. Stern (Yale, USA).

\section{Microscopy and Statistical Analysis}

The method used for immunofluorescence experiment was essentially as described by Ruvkun and Giusto [26]. Animals were fixed in a mixture of $1 \%$ paraformaldehyde, $20 \%$ methanol, permeabilized in $1 \%$ triton. Fixed animals were then incubated overnight with a 1:400 dilution of antiGFP antiserum (Roche), washed extensively and then incubated for another two hours in a 1:500 dilution of the antimouse antibody conjugated to Texas Red (Molecular Probes). After extensive washing, the animals were mounted in DAPI-containing glycerol solution. In some experiments, DiI (Molecular Probes) dye filling was performed on living preparations of worms as previously described [27] to identify chemosensory neurons [28].

To analyze phenotypes, worms were collected on agar pads, anesthetized with levamisol and observed on a Zeiss LSM 510 laser scanning microscope equipped with Normasky optics. The number of $s 2887$ transformed strains analyzed was: 2 for the $P_{\text {let } 756:: l e t-756: \because g f p \text { construct, } 3 \text { for }}$

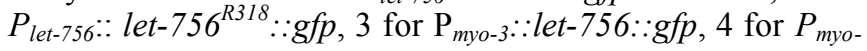
$2: \because$ let-756::gfp and 2 for $P_{\text {egl-17 }}: \because$ let-756. Three $s 2613$ strains transformed by $P_{\text {let-756 }}:$ let-756::gfp were analyzed. In the wild type context, 35 [rol] strains were compared to 2 strains transformed with $P_{\text {let-756 }}:$ let-756::gfp and two strains

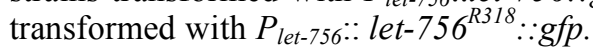

To assess body size in transgenic animals, cultures were synchronized by bleaching gravid animals. Eggs were allowed to grow for 90 hours in the adult stage. Selected plates containing adult rescued [dpy] animals were washed off in
M9 medium and fixed in a mixture of $1 \%$ paraformaldehyde, $20 \%$ methanol. Body sizes (length and width at the vulva) were measured on 100 individual animals using LSM 510 software. Non-parametric Mann-Whitney u test was used. Differences were considered as significant when $p$ values were $<0.01$.

\section{RESULTS}

\section{LET-756 Expression}

To determine where let-756 is required to insure worm viability, we examined the cells that express a GFP reporter from $\sim 4 \mathrm{~kb}$ of promoter and enhancer sequences of let-756

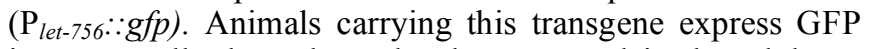
in many cells throughout development and in the adult, as reported by us and others $[7,11,14]$.

The major site of let-756 expression was found in descendant cells of mesodermic precursors (muscle cells). However, some cephalic neurons, CAN cells, and the two glandular cells G1 and G2 (Fig. 1A) were also positive.

Because LET-756 is secreted [23], we expected to find the FGF at some distance from its site of synthesis. We compared the sites of expression to those of LET-756 action as revealed by the localization of a LET-756::GFP fusion. We used a construct where identical let-756 upstream sequence drives the LET-756::GFP fusion protein. We found accumulation of LET-756::GFP mainly in body wall muscle cells and incidentally in vulva, sphincter and pharyngeal muscle cells. However, under microscope observation only few animals were positive for LET-756::GFP despite the fact that they were perfectly rescued; in addition, the fluorescence was often lost with time in rescued strains although the rescuing ability was not.

To correlate protein level expression with rescue capacity we performed immunofluorescence staining of the rescued animals with anti-GFP antibody. Immunodetection of GFP is more sensitive than the observation of its direct fluorescence when exposed to UV light and thus more suitable to detect small amounts of protein. Immunostaining was seen in all rescued individuals, even in the absence of the GFP direct fluorescence, indicating that only animals containing a small number of arrays were able to survive and that these arrays did not contain enough copies of GFP to allow visualization by direct fluorescence.

After GFP immunostaining, animals displayed accumulation of fluorescence in a punctuate pattern. It was not possible to discriminate between a localization of LET-756 at the cell membrane on the cytoplasm side or, more likely, extracellularly on the basement membrane of body wall muscle cells (Fig. 1B,C) and around the pharynx (Fig. 1D), in regions where collagen [29] and UNC-52/perlecan [30] accumulate.

The fusion was observed in the nucleus and not in the nucleolus of CAN cells (Fig. 1E), in agreement with the results obtained with Cos-1 transfected mammalian cells [25]. A construct that mimicked the partial loss-of-function allele, which we have previously denominated LET- $756^{\mathrm{R} 318}$, fused to GFP and under the control of the same upstream sequence, was expressed in muscle cells. Surprisingly, it was also expressed in some neurons exhibiting peculiar endings unable to be filled by the DiI lipophilic dye (Fig. 1F), which 

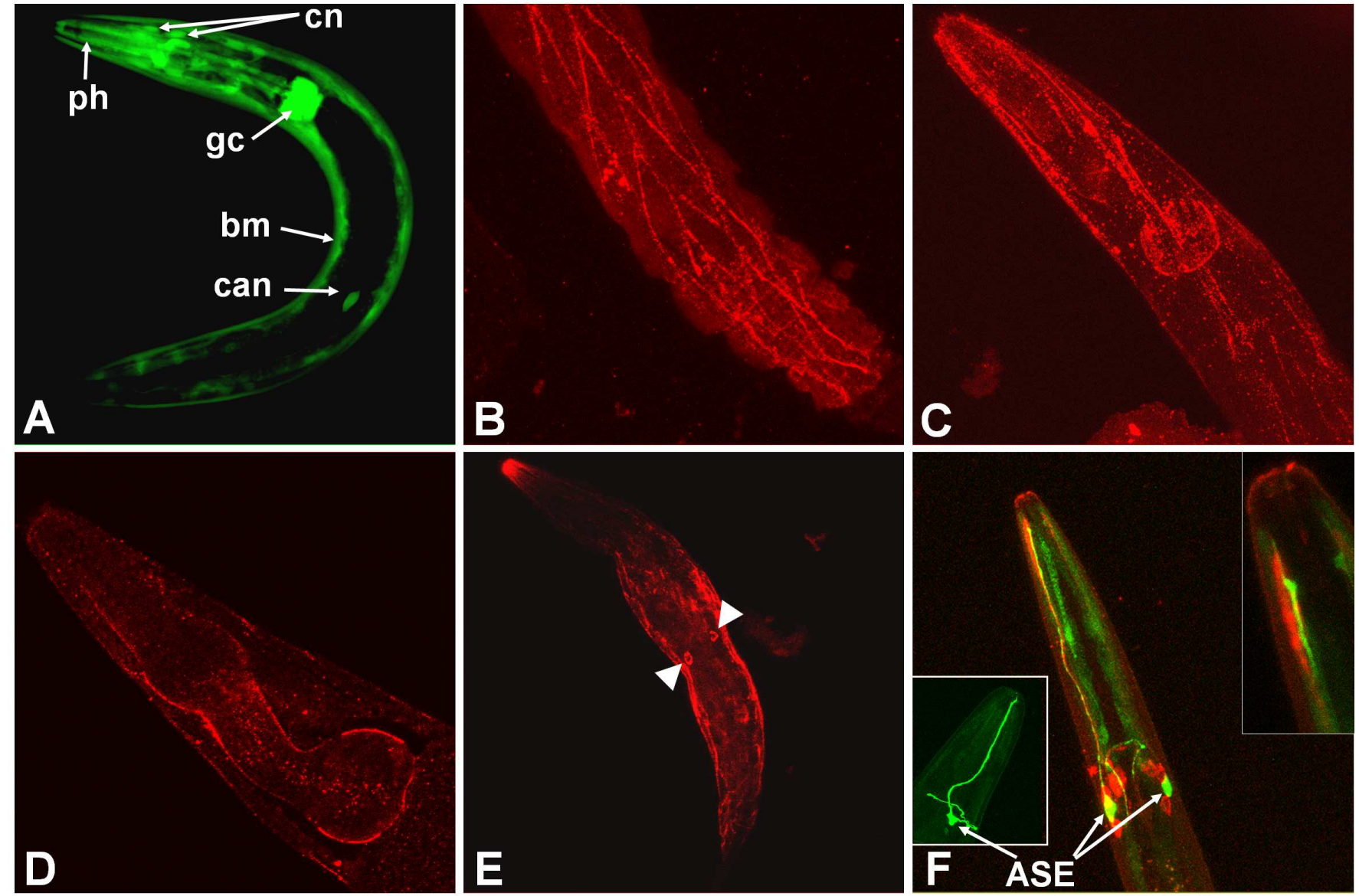

Fig. (1). LET-756 expression in wild type and $l e t-756^{R 318}$ transformed worms. (A) In this mosaic worm, GFP driven from the let-756 promoter is expressed in body muscles (bm), pharynx procorpus (ph), cephalic neurons (cn), CAN cells (can), and G1 and G2 gland cells (gc). (B - E) worms were transformed by $P_{\text {let-756 }}: l e t-756: \because g f p$, fixed and immunostained with anti-GFP antiserum. Accumulation of fluorescence is observed in a punctuate pattern at the junctions between body muscle cells $(\mathbf{C})$ and around the pharynx (D). CAN cells are stained (E). (F) worms were transformed by $P_{\text {let }-756}: \because$ let $-756^{R 318} \because \because g f p$; the construct was mainly expressed in neurons, which were identified as the ASE chemosensorial neurons since they do not fill with the lipophilic DiI dye and exhibit typical endings (magnified in the right insert).

we tentatively identified as the chemosensorial ASE neurons. We never observed this type of fluorescent cells when using the let-756 promoter driving GFP. One possibility is that this staining reflects the specific translocation of the mutated protein in this cell type, but we do not have any definite explanation for this observation. Unlike cells with wt FGF, the nucleolus was also stained in these cells.

\section{Lethality is Rescued by Tissue-Specific Expression of LET-756}

let-756 is expressed mainly in various muscle cells. To determine which of these locations are essential for viability, we created transgenic animals expressing let-756 in various muscles. Constructs were made with tissue-specific promoters and the let-756::gfp C-terminal fusion. For body wall muscle expression we used $P_{\text {myo-3 }}: \because$ let-756 [24] and for pharyngeal expression, we used $P_{\text {myo-2 }}: \because$ let-756 [24]. For sex myoblast expression, we used $P_{\text {egl-17 }}:$ let-756 [9], which drives expression specifically in primary fate vulval precursors and their descendants. These constructs were introduced in animals carrying the let-756(s2887) null allele [dpy17(e164) let-756(s2887) unc-32(e189)] and animals were examined for both rescue activity and phenotype. In addition, we compared the rescuing activity of the wt LET-756 molecule with its truncated form LET $-756^{\mathrm{R} 318}$, which mimics the partial loss-of-function allele $(s 2613)$.

All types of tested ectopic muscle expression rescued the lethal phenotype: 7,6 and 3 strains were obtained using myo3, myo-2 and egl-17 promoters respectively, indicating that muscle expression is sufficient to insure viability.

\section{Worm Body Growth Depends on Spatial Expression of LET-756}

Variations in body length and proportions were observed in the various rescued animals described above. To identify the action of spatially-restricted gene expression on body length and proportions we analyzed transgenic animals with wt let-756 expression driven by both endogenous and heterologous promoters in the null allele ( $s 2887)$ background. Three types of spatial expression patterns were studied: two spatially-restricted expression patterns (anterior and central regions of the worm body, corresponding to the expression of the transgene under the control of the heterologous promoters $P_{m y o-2}$ and $P_{\text {egl-17 }}$, respectively) $[24,9]$ and a widespread expression pattern corresponding to the expression of the transgene under the control of the endogenous and $P_{m y o-3}$ promoters [24]. 
Table 1. Morphometric Analysis of the let-756 Rescued s2887 Allele

\begin{tabular}{|c|c|c|c|c|c|c|c|c|c|}
\hline Genotype & Transgene & $\begin{array}{l}\text { Expression } \\
\text { Sites }\end{array}$ & $\begin{array}{l}\text { Number of } \\
\text { Rescued } \\
\text { Strains }\end{array}$ & $\begin{array}{c}\text { Length } \\
(\mu \mathrm{m})\end{array}$ & $\begin{array}{c}\text { Anterior } \\
(\mu \mathrm{m})\end{array}$ & $\begin{array}{c}\text { Width } \\
(\mu \mathrm{m})\end{array}$ & $\frac{\text { Length }}{\text { Width }}$ & $\frac{\text { Anterior }}{\text { Length }}$ & $\begin{array}{l}\text { Number of } \\
\text { Analysed } \\
\text { Individuals }\end{array}$ \\
\hline $\begin{array}{l}\text { dpy-17(e164) unc- } \\
32(\text { e189) }\end{array}$ & - & - & - & $776 \pm 17$ & $408 \pm 11$ & $82 \pm 2$ & 9.46 & 0.53 & 96 \\
\hline $\begin{array}{l}\text { dpy-17(e164) let- } \\
756(s 2887) \text { unc- } \\
32(e 189)\end{array}$ & $P_{\text {let }-756} \because:$ let -756 & $\begin{array}{l}\text { muscles, } \\
\text { neurons }\end{array}$ & $>20$ & $\begin{array}{c}1029 \pm \\
27\end{array}$ & $603 \pm 15$ & $105 \pm 3$ & 9.80 & 0.59 & 93 \\
\hline $\begin{array}{l}\text { dpy-17(e164) let- } \\
756(s 2887) \text { unc- } \\
32(e 189)\end{array}$ & $P_{\text {let-756: }}: \operatorname{let} 756^{R 318}$ & $\begin{array}{l}\text { muscles, } \\
\text { neurons }\end{array}$ & 5 & $822 \pm 18$ & $425 \pm 11$ & $79 \pm 2$ & 10.40 & 0.52 & 97 \\
\hline $\begin{array}{l}\text { dpy-17(e164) let- } \\
756(s 2887) \text { unc- } \\
32(e 189)\end{array}$ & $P_{m y o-2}:$ let-756 & pharynx & 6 & $745 \pm 19$ & $520 \pm 16$ & $98 \pm 3$ & 7.60 & 0.70 & 140 \\
\hline $\begin{array}{l}\text { dpy-17(e164) let- } \\
756(\text { s2887) unc- } \\
32(e 189)\end{array}$ & $P_{m y o-3} \because$ let-756 & $\begin{array}{l}\text { body } \\
\text { muscles }\end{array}$ & 7 & $920 \pm 21$ & $529 \pm 16$ & $93 \pm 3$ & 9.89 & 0.58 & 126 \\
\hline $\begin{array}{l}\text { dpy-17(e164) let- } \\
756(\text { s2887) unc- } \\
32(\text { e189) }\end{array}$ & $P_{\text {egl-17}} \div:$ let-756 & $\begin{array}{c}\text { sex } \\
\text { myoblasts }\end{array}$ & 3 & $898 \pm 36$ & $477 \pm 18$ & $109 \pm 7$ & 8.24 & 0.53 & 71 \\
\hline
\end{tabular}

Data are presented by mean \pm SE. Body lengths and widths were determined in adult hermaphrodites at 4 days after hatching.

Body sizes of animals were measured - without assessing the size of individual cells - in these different conditions. Individual total body length, mouth-to-vulva length, and width at the vulva are shown in Table 1 .

Expression of wt let-756 from the myo-3 promoter produced animals with the same characteristics as those rescued by using the let-756 promoter (Fig. 2B,E). MYO-3 is expressed in body wall muscles, which are distributed all along the body from head to tail. Therefore, LET-756 expression in body wall muscles is sufficient for full rescue of the lethal phenotype and insures the same body length development as expression driven by the proper promoter. The respective proportions of anterior and posterior body parts in strains rescued with $P_{\text {let-756 }}$ and $P_{m y o-3}$ promoters being similar (Fig. $3)$, the contribution of pharyngeal LET-756 expression seems to be minor.

Expression of LET-756 from the $P_{m y o-2}$ promoter, which drives expression exclusively in the pharynx musculature, led to shorter animals than the one described above. In addition, they exhibited a misplaced vulva due to a better development of the anterior (defined as the length from mouth to vulva) than the posterior part of the body (Fig. 2D). These animals exhibited a ratio "anterior length over total" larger than control individuals or individuals with LET-756 or LET-756 ${ }^{\mathrm{R} 318}$ expression driven by $P_{\text {let-756 }}$ and $P_{m y o-3}$ (Table 1).

Expression of LET-756 from the egl-17 promoter led to animals with larger width size than control animals (Fig. 2F). EGL-17 is expressed in sex muscles [9]. It is thus coherent that LET-756 expression driven by the egl-17 promoter in the middle of the animal increased locally the size of the animals. In addition, the posterior length was also increased compared to control strain and animals rescued with the let- $756^{R 318}$ transgene. Sex muscles arise from a pair of bilaterally symmetrical sex myoblasts; these cells are born in the posterior body. During larval development of the transgenic animals, enough LET-756 vehiculated by migrat- ing myoblasts may allow the growth of the posterior part of the animals.

\section{Reduced Body Size Results from let-756 ${ }^{R 318}$ Transgene Expression}

We have previously reported that no major morphological and/or behavior differences was observed between lines rescued with $P_{\text {let-756 }}:$ let-756 and $P_{\text {let-756: }}:$ let-756 ${ }^{R 318}$ constructs [25], which mimic wt and partial-loss-of-function alleles, respectively. However, in light of our results on body size we analyzed the length and width of these transgenic animals.

As compared to [dpy-17(e164) unc-32(e189)] animals, which have only two copies of the wt let-756 gene, animals from the $s 2887$ strain (null allele) rescued by let-756 expressed under the control of its own promoter had enlarged body length mostly due to a better development of the anterior part of the animal (Table 1, Fig. 2A,B). In contrast, the body length of animals rescued by let- $756^{R 318}$ was smaller than the length of those rescued by the wt molecule, and was in the range of the body length of the control (dpy-17 (e164) unc-32(e189)) animals (Figs. 2C,3). As already reported [29], we found a four-fold increase in the number of transgene copies in let-756 $6^{R 318}$ strains compared to strains rescued with the wt let-756. This increase in array copy number could be explained by different activities of the proteins encoded by transgenes on the tyrosine kinase FGFR/EGL15 , since both FGFs are secreted (23) or by the different subcellular localizations of the two molecules in the expressing cells [25].

Thus, in all circumstances, LET-756 increased the animal body size in areas where it was expressed and body muscle cell expression was sufficient for ensuring normal body length. Quantification indicated that, as compared to [dpy17(e164) unc-3(e189)] animals, the total length of all transgenic animals (except for $P_{\text {myo-2 }}::$ let-756) was significantly increased $(\mathrm{p}<0.01)$ in a hierarchy $P_{\text {let-756 }}:$ let $-756>P_{\text {myo- } 3}: \because$ let- 

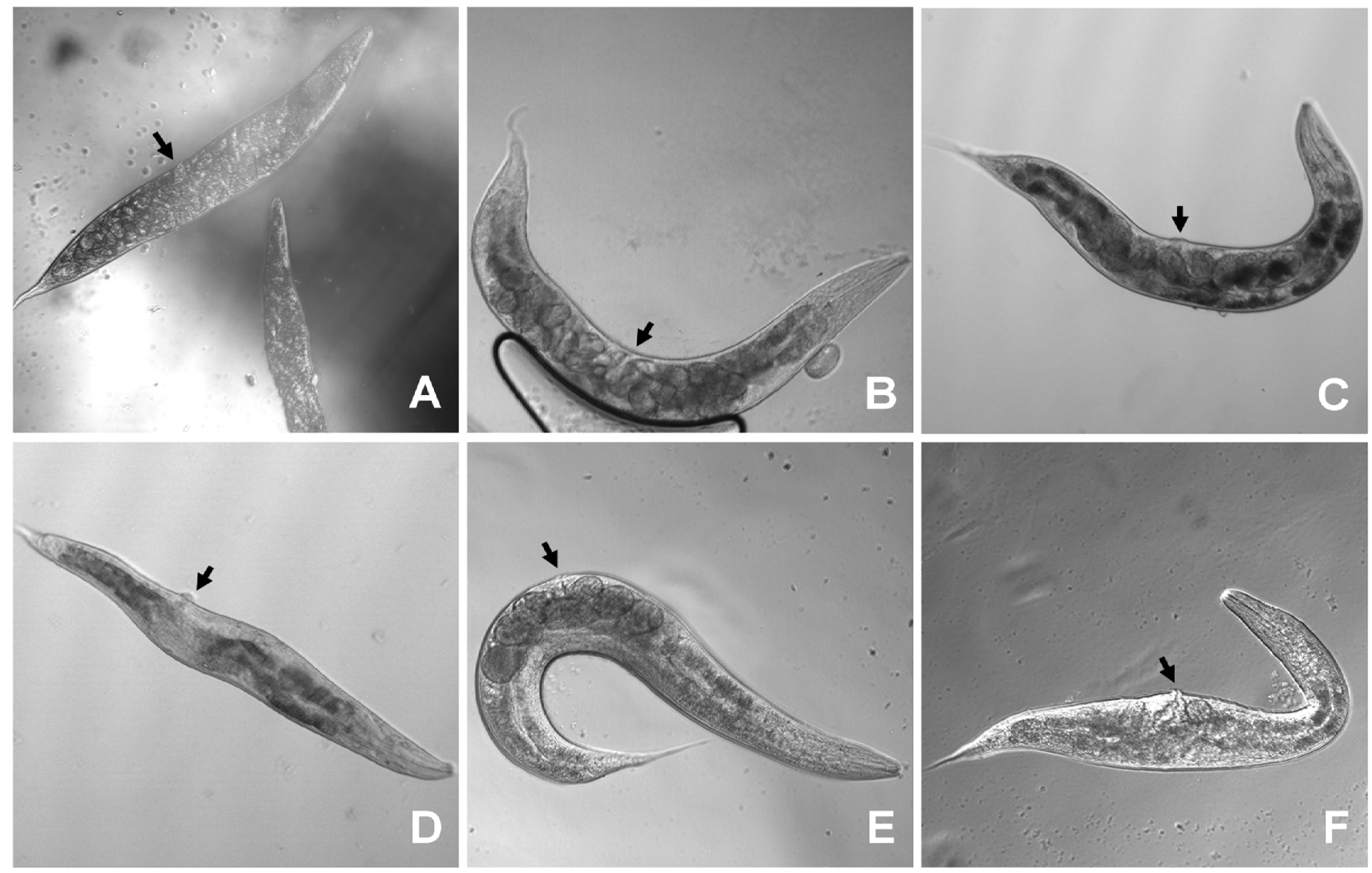

Fig. (2). Worms transformed with let-756 under the control of tissue specific promoters were phenotypically different. Worms transformed by let-756 (B) or let-756 ${ }^{R 318}$ (C) under the control of $P_{\text {let-756 }}$ promoter are not different from the control [Dpy Unc] strain (A) although the former are bigger (see Fig. (3) for morphometric analysis). Worms transformed with let-756 under the control of the $P_{m y o-2}$ promoter show reduction in the size of the posterior part of the body (D). Animals with let-756 under the control of $P_{\text {myo-3 }}$ develop harmoniously (E). Animals with let-756 under the $P_{\text {egl-17 }}$ promoter show enlargement of the body width at the vulva (F). Arrows point to the vulva.

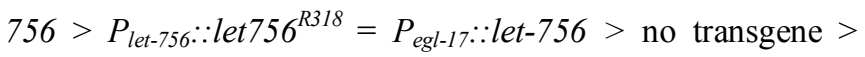
$P_{\text {myo- } 2}: \because$ let-756. In contrast, the anterior part of animals transformed with $P_{m y o-2}: \because$ let-756 was significantly $\left(\mathrm{p}<10^{-5}\right)$ larger than untransformed worms or worms transformed with $P_{\text {egl- }}$ ${ }_{17}: \because$ let-756 or $P_{\text {let-756 }}: \because$ let $756^{R 318}$ transgene. It was not different from worms transformed with $P_{\text {myo-3 }}:$ let-756 and smaller than those transformed with $P_{\text {let-756 }}: \because$ let-756. The posterior part of $P_{\text {myo-2 }}:$ let-756 was shorter than all the other transgenic or wild-type strains $\left(\mathrm{p}<10^{-7}\right)$. Finally, the width of $P_{\text {myo-2 }}: \because$ let-756 and $P_{\text {egl-17 }}: \because$ let-756 transgenic strains was increased $\left(\mathrm{p}<10^{-4}\right)$ as compared to all other animals (Fig. 3).

\section{let-756 Effect on Body Length Does Not Depend on Ge- netic Background}

Some genes coding for cuticle collagens modify worm body length [20]. We were thus concerned by a possible effect of $d p y-17$ (20), which was used as a balancer in the establishement of homozygote strain for let-756 loss-offunction allele, and rol-6 [31], a largely used coinjection marker of transformation, both encoding cuticle collagens.

Due to early lethality of the let-756 loss-of-function allele, we could not compare the size of rescued vs unrescued animals. We thus compared the ratio between body length and width in the various rescued strains, as a hallmark of the
Dpy phenotype. Dpy mutants are defined as small, fatty worms and thus display a larger width to length size ratio than wt animals. The body dimensions were increased in the rescued strains expressing the wt let-756 transgene under the control of the $P_{l e t-756}$ or $P_{m y o-3}$ compared to the $d p y-1((e 164)$ unc-3(e189) reference strain, but, importantly, the width to length size ratio was not modified (Table 1). Thus, overexpression of let-756 cannot suppress the Dpy phenotype of the dpy-17 mutant allele.

To eliminate unambiguously any effect of the $d p y-17$ background on the LET-756-induced body length, we compared the consequence of let-756 transgene expression under the control of its own promoter in the [let-756(s2613) unc$32(e 189)]$ hypomorph as well in wt N2 animals. In the hypomorph, the myo-3 marker was used as an indicator of which area of the body was transformed, and in wt N2 animals, the rol-6 marker was used to identify transformed animals. Expression of LET-756 increased both length and width in the [let-756(s2613) unc-32(e189)] hypomorph in which the $d p y-17$ mutation had being outcrossed (Table 2). As compared to the hypomorph (Fig. 4A), the rescued animals appeared larger and developed a normal number of eggs (Fig. 4B). They did not look different from the unc-32 (e189) strain (Fig. 4C), which contains two wt let-756 al- 


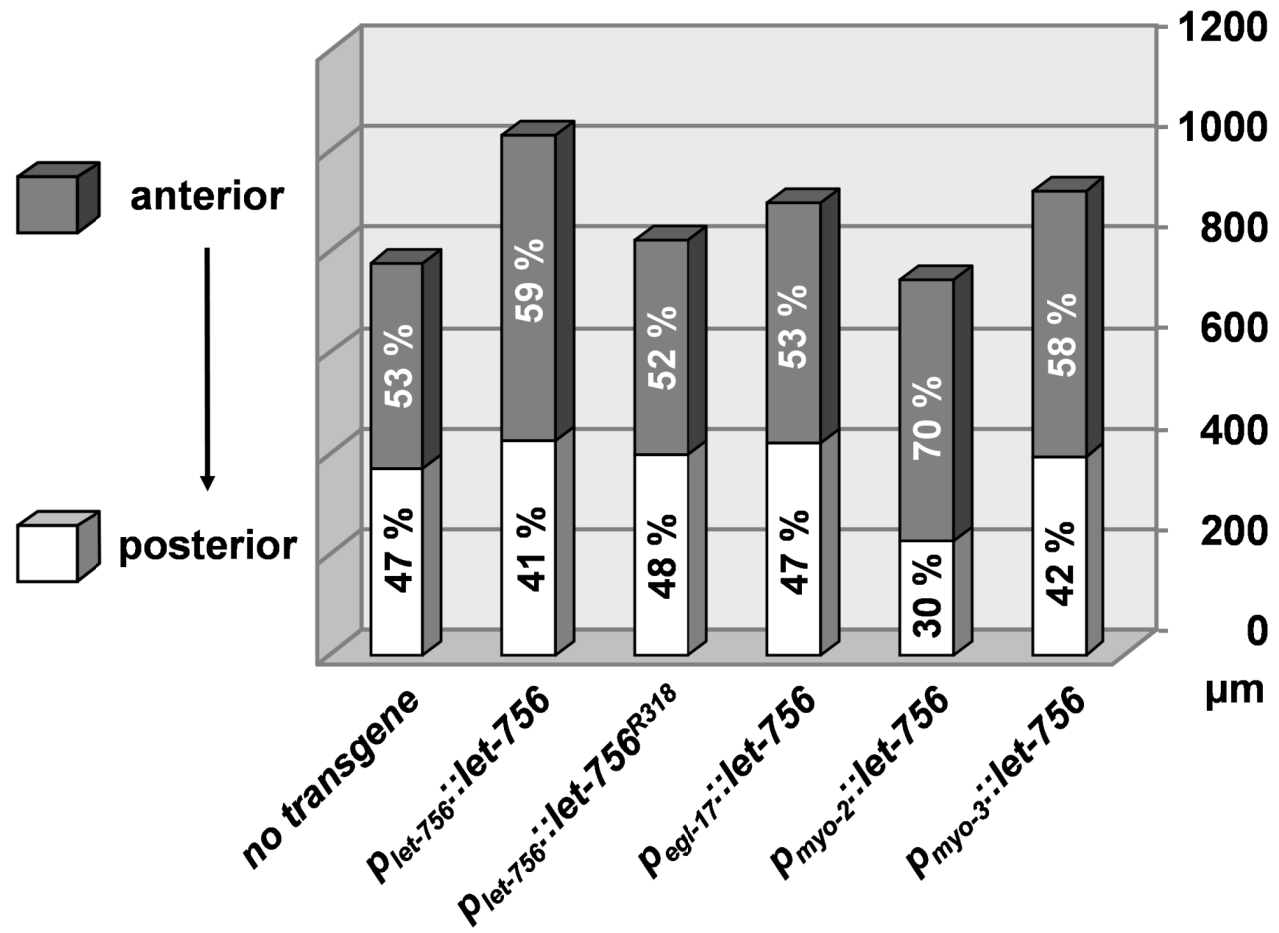

Fig. (3). Morphometric analysis. Anterior (shaded area) and posterior (unshaded) sizes of the different strains are represented (in $\mu \mathrm{m}$ ) as well as their relative contribution in the total body length.

leles. However, in mosaic animals where LET-756 was expressed only in the anterior part of the animal, the posterior part looked scrawny and thinner than normal (Fig. 4D). Reciprocally, animals where FGF expression occurred only in the posterior part of the animals exhibited a thinner anterior part (Fig. 4E,F). Thus, the same phenotypes as those described in the worms carrying the loss-of-function allele s2887 were observed in the animals carrying the partial lossof-function allele $s 2613$, showing that spatial expression of LET-756 increases body size independently of the genetic background.

Finally, we examined the effect of let-756 overexpression in a wt context. We found that $\mathrm{N} 2$ animals injected with with $P_{\text {let-756 }}: \because$ let-756 or $P_{\text {let-756 }}:$ let $756^{R 318}$ were not different in size (Table 3) than those injected with $P_{\text {let- }}$ $756: \because g f p$ although smaller than untransformed worms, due to the presence of rol-6 (su1006) allele, the transformation marker gene. Thus, overexpression of LET-756 or LET$756^{\mathrm{R} 318}$ in a wt context did not result in an overgrowth phenotype. A threshold ligand amount expressed by endogenous alleles of the N2 worms and sufficient to saturate the EGL15 receptors may explain size conservation.

\section{DISCUSSION}

Body size control is an oligogenic/polygenic character that depends on both nutrition-dependent and independent pathways. Studies in Drosophila melanogaster and Caenorhabditis elegans highlighted the insulin pathways as the principal player in controling nutrition-dependent growth rates [32 and 33 for reviews]. In the nematode worm, three nutrition-independent pathways (a TGF $\beta$ pathway, a spectrin pathway and a calcineurin pathway) determine body length by acting on the hypodermis, the primary site of growth regulation. The size of hypodermal cells and the composition of cuticle, the exoskeleton surrounding the body of the worm synthesized by hypodermal cells, are the main factors affecting body size.

In this study, we demonstrate that the FGF pathway is implicated in the control of the worm body size. Although let-756 loss-of-function allele ( $s 2887)$ is lethal, individuals carrying the partial loss-of-function allele (s2613) grow very slowly into small transparent, starved-looking adults. Explanations could be defects in cells that make cuticle or dysfunction of the pharyngeal musculature.

Table 2. Morphometric Analysis of the let-756 Rescued s2613 Allele

\begin{tabular}{|c|c|c|c|c|c|c|}
\hline Genotype & Transgene* & $\begin{array}{c}\text { Number of } \\
\text { Rescued Strains }\end{array}$ & $\begin{array}{c}\text { Length } \\
(\boldsymbol{\mu m})\end{array}$ & $\begin{array}{c}\text { Anterior } \\
(\boldsymbol{\mu m})\end{array}$ & $\begin{array}{c}\text { Width } \\
(\boldsymbol{\mu m})\end{array}$ & $\begin{array}{c}\text { Number of Analysed } \\
\text { Individuals }\end{array}$ \\
\hline \hline unc-32(e189) & - & - & $1074 \pm 29$ & $571 \pm 18$ & $71 \pm 2$ & 37 \\
\hline let-756(s2613) unc-32(e189) & - & - & $790 \pm 11$ & $395 \pm 7$ & $52 \pm 1$ & 52 \\
\hline let-756(s2613) unc-32(e189) & $P_{\text {let-756: }: \text { let-756 }}$ & 7 & $1080 \pm 11$ & $562 \pm 6$ & $78 \pm 1$ & 78 \\
\hline
\end{tabular}



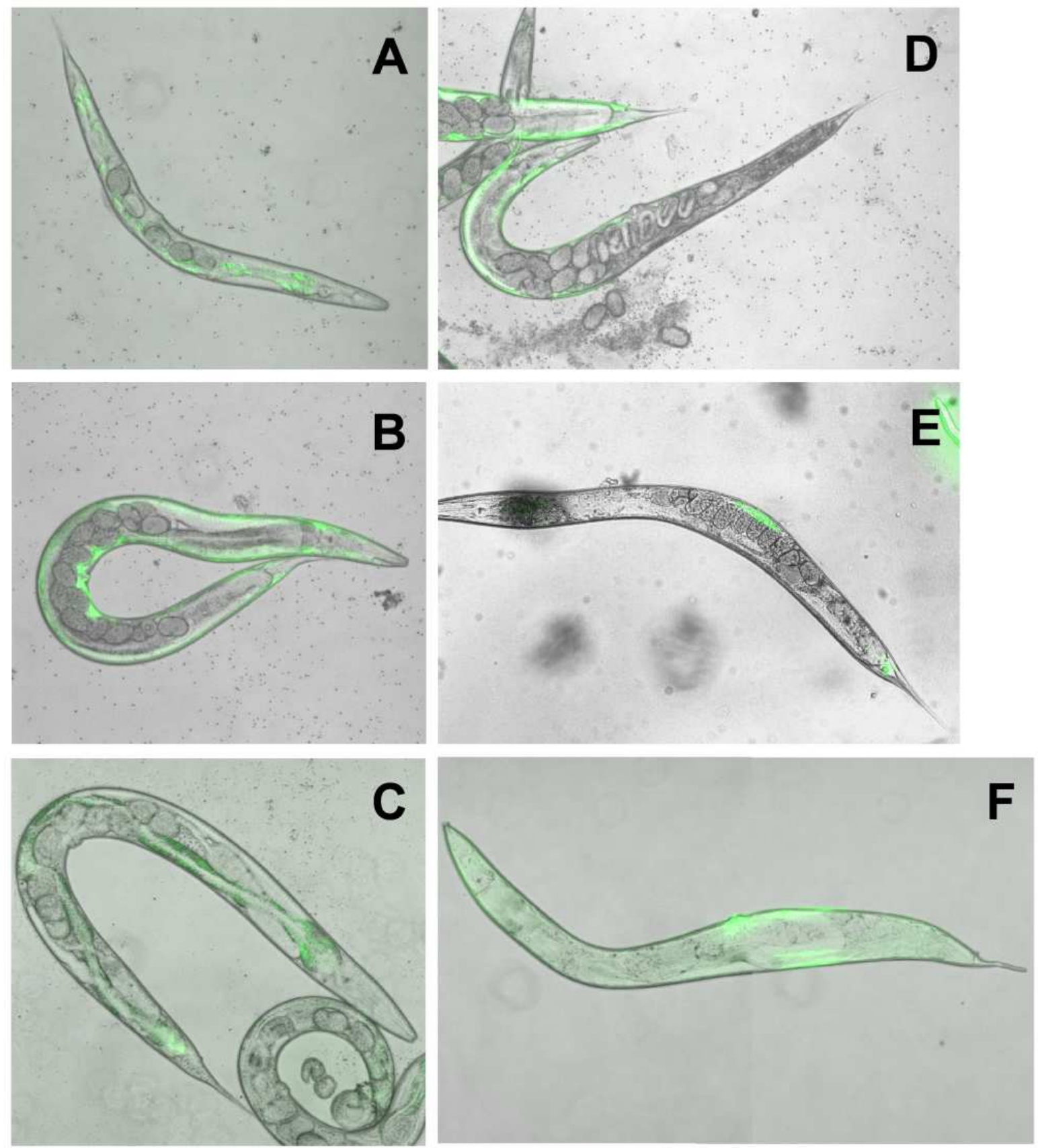

Fig. (4). Rescue of the hypomorph by let-756 expressed under the control of its own promoter. let-756(s2613) unc-32(e189) worms (A) were transformed by the $P_{\text {let-756: }}:$ let-756 construct. (B) shows the phenotype of animals rescued by let-756 expressed in all body muscles. These animals resemble the $u n c-32$ strain (C). Animals shown in (D) exhibit LET-756 expression in anterior muscles: development of the posterior part of the animal is impaired and reciprocally, animals shown in (E) and (F) exhibit LET-756 expression in posterior muscles and their anterior part is thinner than normal. The autofluorescence of the gut granules is visible in (A) and (C).

To determine the focus of lethality and to understand the defect in body size we analyzed which let-756-expressing cell was responsible for correct larval development. We focused on the role of different let-756 expressing muscle cells in allowing post-embryonic development rescue and found that expressing let-756 under the control of the myo-3 promoter induced the same harmonious development of the animal as expressing the endogenous protein under the control of the proper promoter. Body length is controled by hypodermal cells, which make cuticle. Hypodermal cells express EGL-15, the FGF receptor. Most probably, LET756, through paracrine expression in muscle cells, acts on the hypodermal cells, triggers EGL-15 signaling, which regulates fluid balance [12] and cuticle formation. Thus, muscle expression of LET-756 is sufficient to obtain a well developed worm and may be essential for proper development of the nematode $C$. elegans. It is worth noting that the promoter region of let-756 contains a muscle-specific responsive element also present in the myo-3 but not myo-2 promoter [34]. 
Table 3. Morphometric Analysis of the let-756 Surexpression in Wild-Type Animals

\begin{tabular}{|c|c|c|c|c|}
\hline Genotype & Transgene* & Number of Strains & Length $(\boldsymbol{\mu m})$ & Number of Analysed Individuals \\
\hline \hline $\mathrm{N} 2$ & - & 5 & $1330 \pm 15$ & 42 \\
\hline $\mathrm{N} 2$ & $P_{\text {let }-756:}: G F P$ & 6 & $1157 \pm 21$ & 87 \\
\hline $\mathrm{N} 2$ & $P_{\text {let-756: }: \text { let }-756}$ & 11 & $1233 \pm 13$ & 47 \\
\hline $\mathrm{N} 2$ & $P_{\text {let-756: }: \text { let } 756^{R 318}}$ & 11 & $1243 \pm 18$ & 66 \\
\hline
\end{tabular}

*The pRF4 construct containing the rol-6 (su1006) was coinjected as marker.

Another explanation for the impaired growth of the let756 mutant could be a defect in the pharyngeal musculature, impairing the pumping or grinding of bacteria - the nematode food source - or in pharyngeal gland cells. The function of the latter cells is unknown; their anatomy indicates that they are secretory cells, pouring their content in the lumen of the pharynx. Pharynx and gland cells are two sites were LET-756 is expressed. Driving LET-756 expression in the pharyngeal musculature from the myo-2 promoter confers viability to the null allele but the rescue looked incomplete since only the anterior part of the animal developed correctly. Thus, we can exclude a pharyngeal cell-autonomous action of LET-756 responsible for an either mechanistic or digestive problem of feeding. Interestingly, body width was increased in worms transformed by $P_{m y o-2}: \because$ let-756. However, these animals appeared very often [Egl], indicating that the limited available amount of LET-756 at middle body (resulting from the pharynx muscle cells expression) could interfere with EGL-15 activation and not allow proper sex myoblast positioning and/or progeny formation (uterine and vulval muscles). Thus, LET-756 may constitute, alone or in cooperation with other diffusible ligands, the gonadindependent attraction signal for sex myoblast cells described previously [9].

Body length was increased when let-756 was expressed under the control of the egl-17 promoter. This was mainly due to a well-developed posterior part of the animal. EGL17 , the other C. elegans FGF, is expressed in the developing gonad and vulva and acts as the chemoattractant that guides the migration of the sex myoblasts. During sex muscles migration from the posterior body, enough LET-756 vehiculated by migrating myoblasts may allow the growth of the posterior part of the animal. In addition, body width was increased indicating again that LET-756 increases body mass wherever it is produced.

Thus, any spatial rescue results in a local growth of the animal leaving the other part of the animal with a scrawny phenotype. This may result from either abnormal cell growth or from worm lacking sufficient fluid as described by Huang and Stern [12], as observed in the null or partial loss-offunction allele. We suggest that overexpression of LET-756 from a small number of cells - and herein from any cells of mesodermal origin - might be sufficient to regulate fluid balance and trigger EGL-15 activity on hypodermal cells.

Our observations suggest that wherever LET-756 is expressed and acts on its receptor on hypodermal cells, postembryonic development is insured. The extracellular matrix components are essential players in the regulation of local concentration of growth factors and morphogens. Experimental observations showed that glypicans, cell-anchored heparan sulfate proteoglycans, bind growth factors such as FGF in Drosophila [35] and TGF $\beta$ in C. elegans [19], modulating their local concentration and protecting them from proteolytic degradation. We have observed accumulation of LET-756 around the pharynx and in the extracellular space of body muscle cells. Collagens are critical for the assembly and function of the extracellular matrix. It is noteworthy that we found COL-129, the product of a structural collagen gene, interacting with LET-756 in yeast two-hybrid screens [36].

Dixon et al. [14] reported that expression of LET-756 in a single cell is sufficient to rescue the lethality of let756(s2887) null animals and that the rescued animals have a pronounced bulge near the site of expression. Our results indicate that even if the animals are capable of surviving when only one cell type is expressing LET-756, normal body growth is limited to the area where LET-756 can diffuse.

In summary, our data show that misexpression of LET756 , as in the partial loss-of-function allele, reduces the size of the animal as compared to wild-type. Reciprocally, LET756 overexpression in the hypomorph or the null allele increases body size. Expression in body muscles is sufficient to allow the development of the null let-756 allele probably by acting on hypodermal cells through EGL-15 activation. Our results support a juxtacrine/paracrine activity for LET756 exerting locally its effect rather than through a large diffusion through the pseudocoelum.

\section{ACKNOWLEDGEMENTS}

We thank F. Birg and D. Marininchi for encouragements, C. Zemmour for statistical analyses, F. Roubin and M. Monleau for their help at some stages of the study. We are grateful to D. Thierry-Mieg for helpful discussions, M. Stern for the gift of the egl-17 construct, A. Fire for GFP expressing plasmids and the Caenorhabditis Genetics Center (CGC) for strains. The work has been supported by Inserm, Institut Paoli-Calmettes and grants from the Ligue Nationale Contre le Cancer (Label).

\section{REFERENCES}

[1] Goldfarb M. Functions of fibroblast growth factors in vertebrate development. Cytokine Growth Factor Rev 1996; 7: 311-25.

[2] Popovici C, Roubin R, Coulier F, Birnbaum D. An evolutionary history of the FGF superfamily. Bioessays 2005; 27: 849-57.

[3] Olsen SK, Garbi M, Zampieri N, Eliseenkova AV, Ornitz DM, Goldfarb M, Mohammadi M. FHFs share structural but not functional homology to FGFs. J Biol Chem 2003; 278: 34226-36.

[4] Planque N. Nuclear trafficking of secreted factors and cell-surface receptors: new pathways to regulate cell proliferation and differentiation, and involvement in cancers. Cell Commun Signal 2006; 4: 7-18. 
[5] DeVore DL, Horvitz HR, Stern MJ. An FGF receptor signaling pathway is required for the normal cell migrations of the sex myoblasts in C. elegans hermaphrodites. Cell 1995; 83: 611-20.

[6] Goodman SJ, Branda CS, Robinson MK, Burdine RD, Stern MJ. Alternative splicing affecting a novel domain in the C. elegans EGL-15 FGF receptor confers functional specificity. Development 2003; 130: 3757-66.

[7] Birnbaum D, Popovici C, Roubin R. A pair as a minimum: The two fibroblast growth factors of the nematode Caenorhabditis elegans. Dev Dyn 2005; 232: 247-55.

[8] Burdine RD, Chen EB, Kwok SF, Stern MJ. egl-17 encodes an invertebrate fibroblast growth factor family member required specifically for sex myoblast migration in Caenorhabditis elegans. Proc Natl Acad Sci USA 1997; 94: 2433-7.

[9] Burdine RD, Branda CS, Stern MJ. EGL-17(FGF) expression coordinates the attraction of the migrating sex myoblasts with vulval induction in C. elegans. Development 1998; 125: 1083-93.

[10] Roubin R, Naert K, Popovici C, et al. let-756, a C. elegans fgf essential for worm development. Oncogene 1999; 18: 6741-7.

[11] Bulow HE, Boulin T, Hobert O. Differential functions of the $C$. elegans FGF receptor in axon outgrowth and maintenance of axon position. Neuron 2004; 42: 367-74.

[12] Huang P, Stern MJ. FGF signaling functions in the hypodermis to regulate fluid balance in C. elegans. Development 2004; 131: 2595-604.

[13] Szewczyk NJ, Jacobson LA. Activated EGL-15 FGF receptor promotes protein degradation in muscles of Caenorhabditis elegans. EMBO J 2003; 22: 5058-67.

[14] Dixon SJ, Alexander M, Fernandes R, Ricker N, Roy PJ. FGF negatively regulates muscle membrane extension in Caenorhabditis elegans. Development 2006; 133: 1263-75.

[15] Savage-Dunn C. TGF- $\beta$ signalling. WormBook, ed. The C. elegans Research Community, WormBook, doi/10.1895/wormbook.1.22.1 http://www.wormbook.org. 2005

[16] Savage-Dunn C, Maduzia LL, Zimmerman CM, et al. Genetic screen for small body size mutants in $C$. elegans reveals many TGF- $\beta$ pathway components. Genesis $2003 ; 35: 239-47$.

[17] Kuhara A, Inada H, Katsura I, Mori I. Negative regulation and gain control of sensory neurons by the C. elegans calcineurin TAX-6. Neuron 2002; 33: 751-63.

[18] Bandyopadhyay $\mathrm{J}$, Lee $\mathrm{J}$, Lee $\mathrm{J}$, et al. Calcineurin, a calcium/calmodulin-dependent protein phosphatase, is involved in movement, fertility, egg laying, and growth in Caenorhabditis elegans. Mol Biol Cell 2002; 13: 3281-93.

[19] Gumienny TL, MacNeil LT, Wang H, de Bono M, Wrana JL, Padgett RW. Glypican LON-2 is a conserved negative regulator of BMP-like signaling in Caenorhabditis elegans. Curr Biol 2007; 17: 159-64.

[20] Page AP, Johnstone IL. The cuticle, WormBook, ed. The C. elegans Research Community, WormBook, doi/10.1895/wormbook.1. 138.1, http://www.wormbook.org 2007.
[21] Wood WB and the Community of C. elegans researchers. The nematode Caenorhabditis elegans. Cold Spring Harbor Laboratory press: USA, 1988

[22] Stewart HI, O'Neil NJ, Janke DL, et al. Lethal mutations defining 112 complementation groups in a $4.5 \mathrm{Mb}$ sequenced region of Caenorhabditis elegans chromosome III. Mol Gen Genet 1998; 260: 280-8.

[23] Popovici C, Conchonaud F, Birnbaum D, Roubin R. Functional phylogeny relates LET-756 to FGF9. J Biol Chem 2004; 279: 40146-52.

[24] Okkema PG, Harrison SW, Plunger V, Aryana A, Fire A. Sequence requirements for myosin gene expression and regulation in Caenorhabditis elegans. Genetics 1993; 135: 385-404.

[25] Popovici C, Fallet M, Marguet D, Birnbaum D, Roubin R. Intracellular trafficking of LET-756, a fibroblast growth factor of C. elegans, is controlled by a balance of export and nuclear signals. Exp Cell Res 2006; 312: 1484-95.

[26] Ruvkun G, Giusto J. The Caenorhabditis elegans heterochronic gene lin-14 encodes a nuclear protein that forms a temporal developmental switch. Nature 1989; 338: 313-9.

[27] Popovici C, Isnardon D, Birnbaum D, Roubin R. Caenorhabditis elegans receptors related to mammalian vascular endothelial growth factor receptors are expressed in neural cells. Neurosci Lett 2002; 329: 116-20.

[28] Hedgecock EM, Culotti JG, Thomson JN, Perkins LA. Axonal guidance mutants of Caenorhabditis elegans identified by filling sensory neurons with fluorescein dyes. Dev Biol 1985; 111: 15870 .

[29] Ackley BD, Crew JR, Elamaa H, Pihlajaniemi T, Kuo CJ, Kramer JM. The NC1/endostatin domain of Caenorhabditis elegans type XVIII collagen affects cell migration and axon guidance. J. Cell Biol 2001; 152: 1219-32.

[30] Merz DC, Alves G, Kawano T, Zheng H, Culotti JG. UNC$52 /$ perlecan affects gonadal leader cell migrations in C. elegans hermaphrodites through alterations in growth factor signaling. Dev Biol 2003; 256: 173-86.

[31] Cox GN, Laufer JS, Kusch M, Edgar RS. Genetic and phenotypic characterization of roller mutants of C. elegans. Genetics 1980; 95 : 317-39.

[32] Mirth CK, Riddiford LM. Size assessment and growth control: how adult size is determined in insects. Bioassays 2007; 29: 344-55.

[33] Nelson DW, Padgett RW. Insulin worms its way into the spotlight. Genes Dev 2003; 17: 813-8.

[34] GuhaThakurta D, Schriefer LA, Waterston RH, Stormo GD. Novel transcription regulatory elements in Caenorhabditis elegans muscle gene. Genome Res 2004; 14: 2457-68

[35] Yan D, Lin X. Drosophila glypican Dally-like acts in FGFreceiving cells to modulate FGF signaling during tracheal morphogenesis. Dev Biol 2007; 312: 203-16.

[36] Popovici C, Berda Y, Conchonaud F, Harbis A, Birnbaum D, Roubin R. Direct and heterologous approaches to identify the LET756/FGF interactome. BMC Genomics 2006; 7: 105-15. 